\title{
A Multi-Objective Routing Algorithm Based on Auction Game for Space Information Network
}

\author{
Ligang CONG, Huamin YANG, Yanghui WANG, Xiaogiang DI
}

\begin{abstract}
This paper aims to create a resource-saving method for the routing problem in space information network. To this end, a multi-objective routing algorithm was created based on game theory for space information network. Specifically, the auction game was introduced to solve the routing problem using the delay-tolerating network (DTN) protocol. Considering the topological periodicity of low earth orbit (LEO) satellite network, a typical space information network, the dynamic topological structure was divided into relatively static time slots. Then, the routing problem was solved through the auction game in these slots. The proposed algorithm can minimize the number of selfish nodes in the network and avoid network congestion resulted from excessive resource consumption of individual nodes. Finally, the proposed algorithm was compared with other well-known routing models like the epidemic routing model (Epidemic) and the first contact routing model (FC). The results show that the proposed algorithm outperformed the contrastive models in both average delay and network overhead ratio. The research findings shed important new light on the routing of space information network.
\end{abstract}

Keywords: auction game; multi-objective routing; space information networks

\section{INTRODUCTION}

Despite immense popularity, space information network faces many problems in the construction process, owing to its dynamic topological structure, limited node resources and long communication delay. One of the most prominent problems is routing, a critical step in network construction.

Many plans have been developed to solve the routing problem. Based on finite-state automation, Chang et al. [1] created a routing algorithm that simplifies the computation through the control of topological changes. However, the routing algorithm may worsen the network adaptivity in emergencies and magnify the demand for on-board storage. Ercetin et al. [2] put forward a probabilistic routing protocol (PRP), which reduced the number of times of call interruption and routing redefinition due to routing switching, but increased the call blocking rate and the probability of network congestion. Lee et al. [3] proposed a hierarchical quality of service (QoS) routing protocol (HQRP). On the upside, the HQRP optimizes the switching process and channel resources, thereby reducing the call interruption; on the downside, this protocol increases the overhead and lowers the efficiency of the network.

Jukan et al. [4] proposed a QoS-based distributed routing algorithm: first, the source node sends connection requests to the destination node via flooding, while each intermediate node continuously updates QoS parameters; once the packet reaches the destination node, the link failing to meet QoS requirements is deleted according to each node parameter on the path; finally, the minimum hop number or link lifetime is selected in the path satisfying the conditions, and the longest path is taken as the route. Taleb [5] put forward an explicit load balancing routing algorithm, which selects the route under the known load of the next link, and informs other satellites to slow down data transmission when links are congested. However, this algorithm may fail in the case of severe congestion. Other typical algorithms for satellite routing include agent-based loading balancing routing [6], datagram routing algorithm [7], cross entropy accelerated ant routing [8], etc.
With different emphases, each of the above methods has its own advantages and features in application. In view of this, this paper presents a multi-objective routing algorithm based on game theory for space information network. Specifically, the auction game was introduced to solve the routing problem using the delay-tolerating network (DTN) protocol. Considering the topological periodicity of low earth orbit (LEO) satellite network, a typical space information network, the dynamic topological structure was divided into relatively static time slots. Then, the routing problem was solved through the auction game in these slots. The proposed algorithm can minimize the number of selfish nodes in the network and avoid network congestion resulted from excessive resource consumption of individual nodes. Moreover, it is easy to adapt the routing algorithm to other environments through parameter modification.

With different emphases, each of the above methods has its own advantages and features in application. In view of these, this paper presents a multi-objective routing algorithm based on game theory for space information network. Specifically, the auction game was introduced to solve the routing problem using the delay-tolerating network (DTN) protocol. Considering the topological periodicity of low earth orbit (LEO) satellite network, a typical space information network, the dynamic topological structure was divided into relatively static time slots. Then, the routing problem was solved through the auction game in these slots. The proposed algorithm can minimize the number of selfish nodes in the network and avoid network congestion resulting from excessive resource consumption of individual nodes. Moreover, it is easy to adapt the routing algorithm to other environments through parameter modification.

\section{SYSTEM MODEL}

\subsection{Topological Structure of LEO Satellite Network}

The topological structure of LEO satellite network, a typical space information network, changes continuously and periodically due to the movement of its nodes (satellites) along the pre-set orbits. During the routing of 
this network, it is possible to abstract static models from the dynamic network topology through the control of the topological changes. Relevant strategies mainly include the virtual topology strategy [8], virtual node strategy [10] and division of coverage domains [11].

Here, the virtual topology strategy is adopted to discretize the dynamic topological relationship between network nodes, and divide a complete running cycle of the network into several time slots, denoted as $\left[t_{0}, t_{1}\right],\left[t_{1}, t_{2}\right]$, $\ldots,\left[t_{n}, t_{n}-1\right]$. In each time slot, the network topology is assumed to be constant except at time nodes $t_{1}, t_{2}, t_{3}, \ldots, t_{n}$.

Each satellite in the network stores the status information of itself and that of the neighbouring satellites. The status information is saved in the form of tables, covering communication distance, residual node storage, hop counts to the destination, etc. These tables change periodically with the topological structure of the network. Taking the satellite $v_{1}$ as an example, the status information of itself and its neighbouring satellites within the time slot $\mathrm{t}$ is listed in Tab. 1 below.

Table 1 Information table of satellite $v_{1}$

\begin{tabular}{|c|c|c|}
\hline $\begin{array}{c}\text { Adjacent satellite } \\
\text { nodes }\end{array}$ & $\begin{array}{c}\text { Communication distance } \\
\text { (Unit: km) }\end{array}$ & $\begin{array}{c}\text { Hop counts through } \\
\text { this neighbor node to } \\
\text { the target }\end{array}$ \\
\hline$v_{2}$ & $d_{2}$ & $h_{2}$ \\
\hline$v_{3}$ & $d_{3}$ & $h_{3}$ \\
\hline$v_{4}$ & $d_{4}$ & $h_{4}$ \\
\hline
\end{tabular}

\subsection{Routing Problem}

This research aims to design a mechanism to boost the cooperation between nodes, and thus set up a reliable and efficient spatial route. Based on the DTN protocol, the basic store-and-forward routing mode was adopted for the LEO satellite network, turning the routing problem into the search for the proper store-and-forward nodes.

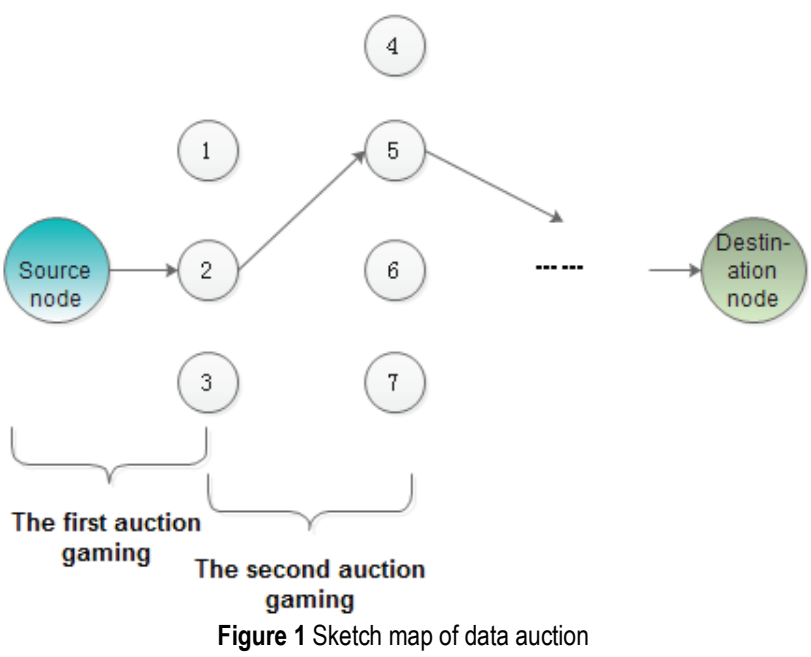

As shown in Fig. 1, multiple paths are available in the network to send the data generated from a node to the destination. The first step of path selection is to determine the suitable forwarding node among the multiple neighbouring nodes. Here, the forwarding node is selected through a process like the item auction. Thus, the selection process can be abstracted as an auction game [14, 15, 17], with the source node as the buyer of forwarding services and its neighbouring nodes as seller of such services.
In general cases, multiple forwarding nodes are required to send the data to the destination, i.e. the auction must be hosted repeatedly. At the end of each auction, the forwarding node should be turned from the seller into the buyer in the next auction, while all the other nodes should be excluded from the next auction. This process should be repeated until the data reach the destination node.

\section{ROUTING AUCTION GAME MODEL}

As mentioned above, the routing problem is abstracted in this paper as an auction game. Thus, this section defines the gain rules of the game and builds the gain models for both the source and forwarding nodes.

\subsection{Gain Rules}

The routing auction game model considers the sending node as the buyer and its neighbouring nodes that have participated in an auction before as sellers. The sellers demonstrate their data forwarding services to the buyer, such that the latter could select the seller with the best service price. All the nodes involved in the data transmission will be compensated by the destination node. To avoid selfish nodes, the success rate was introduced to the data forwarding process. The higher the success rate, the higher the gains, and the inverse is also true. The gain rules of relevant nodes are detailed below:

(1) Three types of nodes are involved in the routing process, namely, source node, forwarding node and destination node.

(2) The gain of any node outside these three types is zero.

(3) The gain of the source node comes from the compensation by the destination node.

(4) The gain of a forwarding node consists of the reception gain and the transmission gain in the auction game.

(5) The destination node has no gain but pays for the services provided by other nodes.

(6) The relevant costs must be deducted to get the final gains of the source and destination nodes.

\subsection{Gain Rules}

Price is the key basis for the selection of forwarding node. In our model, the price of data forwarding services is defined according to link quality and node status. Specifically, the source node selects the node with the lowest bid as the forwarding node. Each bid covers two critical determinants of auction effect: satellite link quality and success rate. The relevant definitions are described as follows.

Definition 1: The link quality of the LEO satellite network can be expressed as:

$r_{i j}(t)=x\left[L_{i j}(t)+h_{j}(t)+\frac{m_{i p}}{m_{j}(t)}\right]$

where $L_{i j}(t)=32.44+20 \log d_{i j}(t)+20 \log f_{i}$ is the spatial propagation loss incurred as the data are transmitted from satellite $v_{i}$ to satellite $v_{j}$ at time $t ; d_{i j}(t)$ is the distance between satellite $v_{i}$ and satellite $v_{j}$ at time $t ; h_{j}(t)$ is the hop 
count for satellite $v_{i}$ to send data to the destination node via satellite $v_{j}$ at time $t ; m_{j}(t)$ is the residual storage of satellite $v_{j}$ at time $t ; m_{i p}$ is the data volume to be transmitted; $r_{i j}(t)$ is the indicator of link quality; $x$ is an adjustment coefficient in the range of $[0,1]$. Note that the hop count depends on the network scale; the link quality indicator is negatively correlated with the actual link quality; the adjustment coefficient, negatively correlated with the actual link quality, aims to optimize the benefits according to the specific situation.

Definition 2: The success rate of data forwarding can be expressed as:

$$
s_{i}(t)=\frac{p_{i s}(t)}{p_{i r}(t)}
$$

where $p_{i s}(t)$ is the total number of data packets sent by satellite $v_{i}$ by the time $t ; p_{i r}(t)$ is the total number of data packets received or generated by satellite $v_{i}$ by the time $t$.

By the above definitions, each bid for forwarding services can be expressed as:

$c_{i j}(t)=\frac{r_{i j}(t)}{s_{j}(t)}$

where $c_{i j}(t)$ is the price offered for forwarding services by satellite $v_{i}$ to satellite $v_{j}$ at time $t$. The price is positively correlated with the link quality and negatively with the success rate.

As mentioned before, the target network adopts the basic store-and-forward routing mode and follows the DTN protocol. The routing cannot be done if the storage of a node is zero or smaller than the data to be received. As a result, the neighbouring nodes with insufficient storage are excluded from bidding.

After all eligible neighbouring nodes place their bids, the source node will select the node with the lowest price for data forwarding. In this case, the source node needs pay the following expense:

$o_{i k}(t)=\min c_{i k}(t) k \in N_{i}$

where $N_{i}$ is the set of neighbouring nodes of node $v_{i}$. The selected node will trade at the price $o_{i}(t)$.

\subsection{Gain Model for Source Node}

The source node acts as the buyer in the auction model for the routing problem. It is assumed that the source node receives relevant compensation from the destination node every time it sends a unit of data, and uses the compensation to pay for the forwarding price. The difference between the compensation and the price is the gain, denoted as g, of the source node.

For simplicity, the cost of data generation and transmission of the source node is assumed to be zero. Then, the gain function of the source node $v_{i}$ can be expressed as:

$$
u_{i}(t)=g \cdot m_{i p}-o_{i}(t) k \in N_{i}
$$

where $m_{i p}$ is the data volume sent by the source node; $g \cdot m_{i p}$ is the total compensation received from the destination node. If $g \cdot m_{i p} \leq o_{i}(t)$, the source node has a negative gain, and will refuse to send data. Therefore, the destination node must provide a suitable amount of compensation according to the importance of the source node. To acquire more gains, the source node will try to improve the success rate as much as possible. This mechanism helps to enhance routing efficiency, relieve network congestion and minimize the selfishness of the satellites.

\subsection{Gain Model for Forwarding Nodes}

Suppose node $v_{j}$, which is not the destination node, is selected for data forwarding. Then, this node can acquire gains in two ways: receiving the forwarding price from the previous node as the seller of forwarding services, and receiving the compensation in the new auction as the buyer of forwarding services.

(1) Gains as the seller

As mentioned before, $c_{i j}(t)$ is the price received by the forwarding node $v_{j}$ for data reception. The received data will take up certain storage of the node, incurring an amount of storage cost. Considering these, the gain function for the node as the seller can be expressed as:

$u_{j 1}(t)=c_{i j}(t)-\frac{m_{j r}}{m_{j}(t)}$

where $m_{j r}$ is the received data volume; $m_{j}(t)$ is the residual storage of the node before the reception.

(2) Gains as the buyer

When the forwarding node $v_{j}$ sends data, it becomes the buyer for forwarding services in a new auction. The new auction has the same procedure as the last auction, except for the absence of the previous node $v_{i}$. Hence, the gain function for node $v_{j}$ as the buyer can be expressed as:

$u_{j 2}(t)=g \cdot m_{j p}-o_{j}(t)$

where $o_{j}(t)$ is the trade price between node $v_{j}$ and its neighbouring nodes in the second auction game; $m_{j p}$ is the volume of data to be sent. Thus, the total gain of node $v_{j}$ in routing is the total gain acquired in data reception and forwarding:

$$
u_{j}(t)=u_{j 1}(t)+u_{j 2}(t)
$$

\section{ANALYSIS AND SIMULATION}

According to formulas in Section 3, the gains of each satellite in the routing process are mainly affected by the spatial propagation loss of electromagnetic waves, node storage and path hop counts. These three factors directly bear on the gains of satellite node, and in turn on the selection of routing nodes. Therefore, this section attempts to disclose the relationships between these factors, node gains and path selection.

As shown in Fig. 2, a local part of the space information network in the time slot $t$ is abstracted as an undirected graph with such six satellites as $v_{1}$ (source 
node), $v_{2}, v_{3}, v_{4}, v_{5}$ and $v_{6}$ (destination node). The line between any two nodes represents an available communication link between the two satellites. If there is no line between two nodes, it means the two satellites cannot communicate with each other directly.

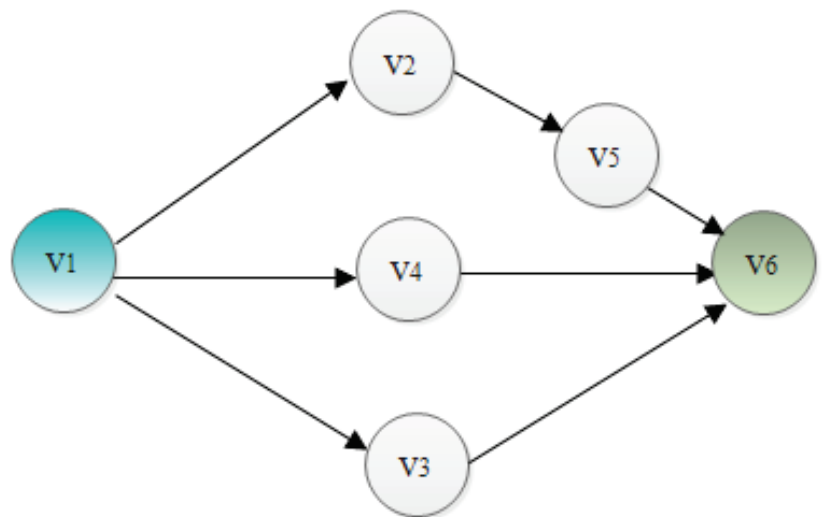

Figure 2 Sketch map of a local part of the LEO satellite network

When node $v_{1}$ generates data and needs to send them to the destination node $v_{6}$, the neighbouring nodes will participate in an auction game. The hop counts of $v_{1}$ to the destination node via $v_{2}, v_{3}$ and $v_{4}$ are 2, 1 and 1 , respectively. Suppose that the data volume of the source node is $100 \mathrm{MB}$. Then, the destination node needs to pay a compensation of $g$ for the transmission of these data. Under these conditions, the author explored the impacts of spatial propagation loss, hop count and residual storage on node gains and path selection.

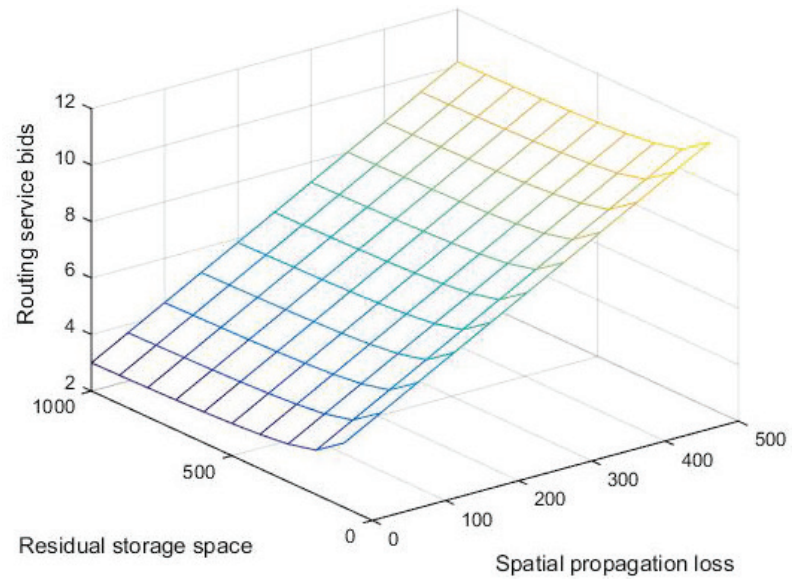

(a) Success rate: $70 \%$

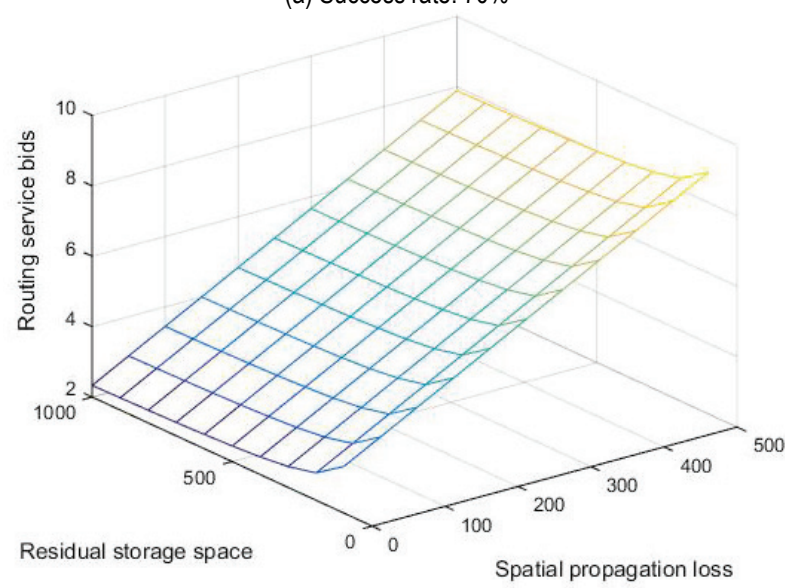

(b) Success rate: $80 \%$

Figure 3 Bid variation of $v_{2}$

\subsection{Analysis of Node Bids}

Suppose the spatial propagation loss and residual storage change in the interval of $[100,500]$ and $[1,10]$, respectively, and that the satellite nodes have two success rates: $70 \%$ and $80 \%$. In this case, the variation in the bids of satellites $v_{2}, v_{3}$ and $v_{4}$ can be determined (Figs. 4 and 5).

As shown in Fig. 3, the bid of node $v_{2}$ increased with the spatial propagation loss but decreased with the growth in residual storage. Under the same conditions, the bid is negatively correlated with the success rate.

With the same hop count, $v_{3}$ and $v_{4}$ changed consistently in their bids for routing services.

It can be seen from Fig. 4 that, under the same hop count, the bid is positively correlated with the residual storage and negatively with the spatial propagation loss. Compared with Fig. 4, it is clear that the hop count has a positive correlation with the bid under the same conditions.

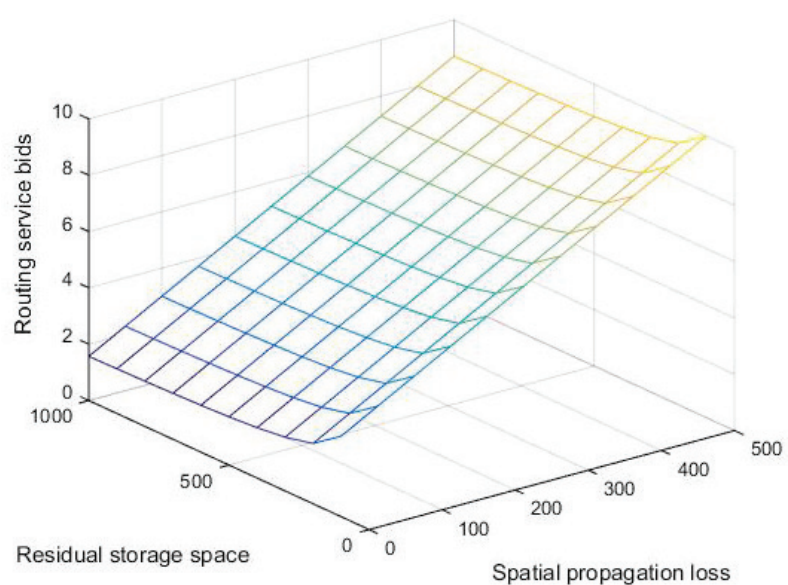

(a) Success rate: $70 \%$

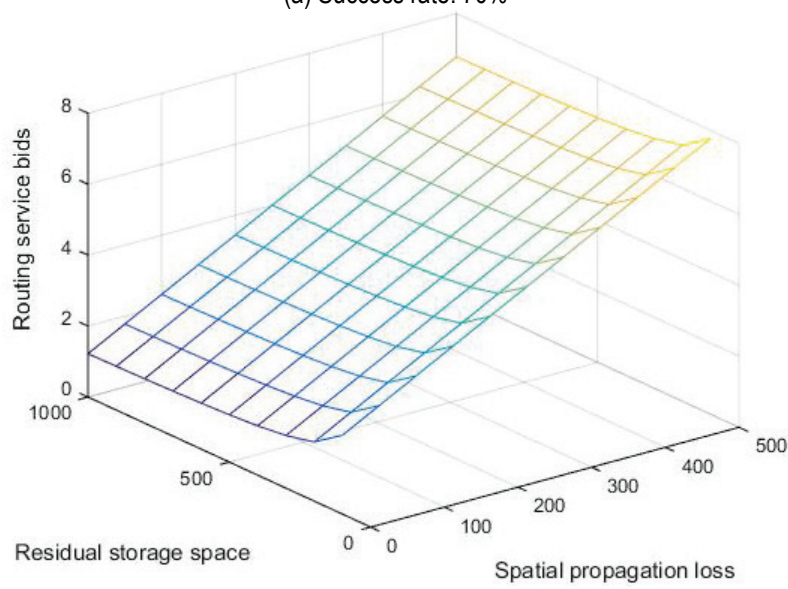

(b) Success rate: $80 \%$

Figure 4 Bid variation of $v_{3}$ and $v_{4}$

\subsection{Analysis on Node Gains and the Routing Process}

The status parameters of $v_{2}, v_{3}, v_{4}$ and $v_{5}$ are listed in Tab. 2. It is assumed that the received data volume equals the sent data volume throughout the transmission between the nodes. Then, the node gains were analysed through a simple simulation of path selection.

As stated above, three paths are available for data transmission from the source node to the destination mode: $v_{1} \rightarrow v_{2} \rightarrow v_{5} \rightarrow v_{6}, v_{1} \rightarrow v_{3} \rightarrow v_{6}$ and $v_{1} \rightarrow v_{4} \rightarrow v_{6}$. In our research, the routing process is converted into the search for the most cost-effective forwarding service. Through 
comparison, the node $v_{1}$ selects the node with the lowest bid for data forwarding, because lower bids mean reduced spatial propagation loss, more residual storage and fewer times of forwarding. Finally, the path $v_{1} \rightarrow v_{4} \rightarrow v_{6}$ was selected after comparing the gains of nodes in different paths (Tab. 3). After repeated auctions, the source node managed to find a suitable path for data forwarding.

Table 2 Status parameters of forwarding nodes

\begin{tabular}{|c|c|c|c|c|}
\hline $\begin{array}{c}\text { Node } \\
\text { Parameter }\end{array}$ & $\begin{array}{c}\text { Free-space } \\
\text { propagation } \\
\text { loss }\end{array}$ & $\begin{array}{c}\text { Residual } \\
\text { storage } \\
\text { space }\end{array}$ & $\begin{array}{c}\text { Hop counts } \\
\text { to the } \\
\text { destination }\end{array}$ & $\begin{array}{c}\text { Data } \\
\text { forwarding } \\
\text { rate }\end{array}$ \\
\hline$v_{2}$ & 220 & 400 & 2 & $80 \%$ \\
\hline$v_{3}$ & 270 & 500 & 1 & $80 \%$ \\
\hline$v_{4}$ & 170 & 400 & 1 & $90 \%$ \\
\hline$v_{5}$ & 170 & 300 & 1 & $80 \%$ \\
\hline
\end{tabular}

Table 3 Gains of nodes in different paths

\begin{tabular}{|c|c|c|c|c|c|}
\hline $\begin{array}{c}\text { Node } \\
\text { GainPath }\end{array}$ & $v_{1}$ & $v_{2}$ & $v_{3}$ & $v_{4}$ & $v_{5}$ \\
\hline $\begin{array}{c}v_{1} \rightarrow v_{2} \rightarrow \\
v_{5} \rightarrow v_{6}\end{array}$ & $g-5.563$ & $g+1.421$ & 0 & 0 & $g+3.359$ \\
\hline $\begin{array}{c}v_{1} \rightarrow v_{3} \rightarrow \\
v_{6}\end{array}$ & $g-4.875$ & 0 & $g+4.375$ & 0 & 0 \\
\hline $\begin{array}{c}v_{1} \rightarrow v_{4} \rightarrow \\
v_{6}\end{array}$ & $g-3.278$ & 0 & 0 & $g+2.928$ & 0 \\
\hline
\end{tabular}

Table 4 Main simulation parameters

\begin{tabular}{|c|c|c|}
\hline $\begin{array}{c}\text { Parameters } \\
\text { rate }\end{array}$ & Value & Description \\
\hline $\begin{array}{c}\text { Node mobility } \\
\text { model }\end{array}$ & MapRouteMovement & $\begin{array}{c}\text { MapRoutMovement } \\
\text { Satellite node mobility } \\
\text { model } \\
\text { MapRoutMovement }\end{array}$ \\
\hline $\begin{array}{c}\text { Node mobility } \\
\text { speed }\end{array}$ & 3,5 & $\begin{array}{c}\text { The mobility speed/second } \\
\text { is } 3-5 \mathrm{~km}\end{array}$ \\
\hline $\begin{array}{c}\text { Node storage } \\
\text { space }\end{array}$ & $1000 \mathrm{MB}$ & $\begin{array}{c}\text { The storage space of } \\
\text { satellite nodes is } 1000 \mathrm{MB}\end{array}$ \\
\hline $\begin{array}{c}\text { Data generation } \\
\text { intervals }\end{array}$ & 8,12 & $\begin{array}{c}\text { A data packet is } \\
\text { generated at every } 8-12 \\
\text { seconds }\end{array}$ \\
\hline Data survival time & 120 & $\begin{array}{c}\text { The survival time of the } \\
\text { data packets is } 60 \text { min }\end{array}$ \\
\hline
\end{tabular}

\subsection{Simulation Experiment}

The proposed routing algorithm was simulated on ONE1.5, a special simulation software for DTN network. The software does not support 3D motion simulation. Thus, the data were pre-processed to convert the $3 \mathrm{D}$ motions of the satellite network into $2 \mathrm{D}$ motions. The preprocessing was implemented through the following steps: First, build a space motion model for the satellite network in Systems Tool Kit (STK); then, project the satellite motion curve in a cycle onto the Earth surface, forming a 2D motion curve; next, convert the 2D motion curve into WKT data through OpenJump before scaling down the model map; finally, build the simulation model using MapRoutMoment. The simulation was carried out on the scene in Fig. 3, which includes six LEO satellites. The main simulation parameters are shown in Tab. 4.

During the simulation, the proposed routing auction game model was compared with the epidemic routing model (Epidemic) and the first contact routing model (FC). (1) Average delay analysis

Fig. 5 compares the average delays of the three models in the simulation. As shown in the Fig. 5, the FC had longer average delay than the constative models. This is because the FC routing algorithm transmits data in single copies, which reduces the probability of data arrival. On the contrary, the Epidemic boasted the smallest average delay, because it generates lots of message copies in the ideal network states. As for the proposed model, its average delay was slightly longer than that of Epidemic but shorter than that of the FC at the start of the simulation. With the elapse of time, the average delay of the Epidemic gradually increased due to the accumulation of message copies. By contrast, the average delay of the proposed model was stable throughout the simulation. Therefore, the proposed model outperformed the other two models in terms of delay.

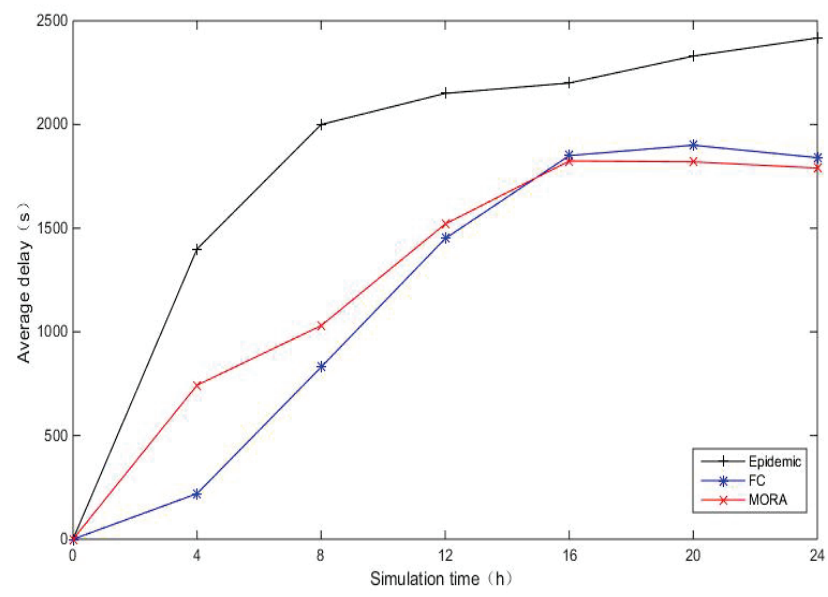

Figure 5 Average delays of the three models

(2) Network overhead ratio analysis

The network overhead ratio refers to the ratio of the data volume transmitted to the destination to the data volume not transmitted to the destination. It is a good indicator of the overall transmission performance of the network.

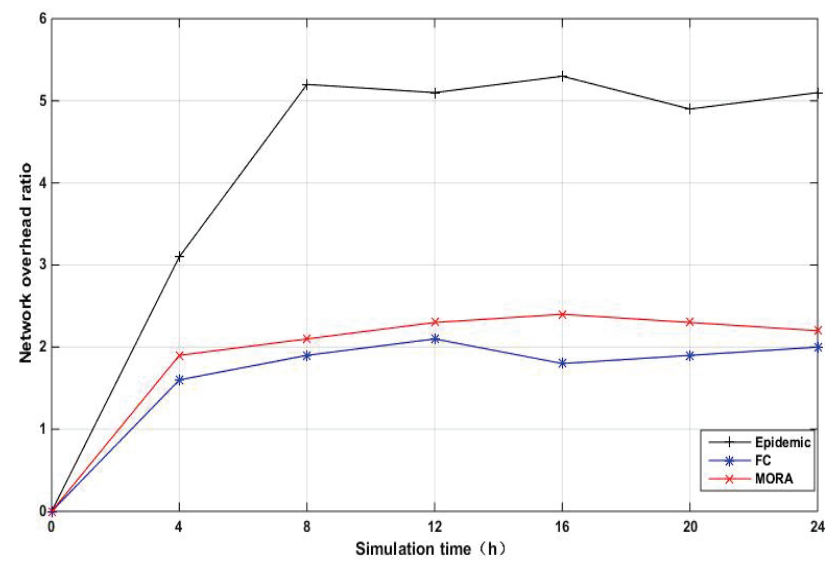

Figure 6 Network overhead ratios of the three models

As shown in Fig. 6, the proposed model achieved a much lower network overhead ratio than the Epidemic and a similar ratio as the FC. The good performance is attributable to the fact that the proposed model neither generates many data copies nor causes severe network congestion. After all, our model was designed to enhance network performance without sacrificing network resources. 


\section{CONCLUSIONS}

This paper creates a routing model based on the auction game for space information networks like the DTN-based LEO satellite network. This type of networks is featured by dynamic topological changes and limited resources. Through simulation, it is learned that the proposed model can realize a shorter average delay, lower consumption of network resource, and better network performance than the Epidemic and the FC. Suffice it to say that the proposed model is a desirable tool for the routing of satellite network. In future research, the proposed model will be compared with other routing algorithms for satellite networks on more simulation tools and will be further improved to determine the optimal path, relieve network congestion and enhance network efficiency.

\section{Acknowledgements}

Supported by the National High-tech R\&D Program of China (2015AA015701); Supported by Foundation of Jilin Educational Committee (JJKH20170628KJ).

\section{REFERENCES}

[1] Chang, H. S., Kim, B. W., Lee, C. G., Min, S. L., Choi, Y., \& Yang, H. S. (1998). FSA-based link assignment and routing in low-earth orbit satellite networks. IEEE Transactions on Vehicular Technology, 47(3), 1037-1048. https://doi.org/10.1109/25.704858

[2] Erçetin, Ö., Krishnamurthy, S., Dao, S., \& Tassiulas, L. (2002). Provision of guaranteed services in broadband leo satellite networks. Computer Networks, 39(1), 61-77. https://doi.org/10.1016/S1389-1286(01)00298-5

[3] Lee, J. \& Kang, S. (2002). Satellite over satellite (SOS) network: a novel architecture for satellite network. IEEE Infocom Conference on Computer Communications Nineteenth Joint Conference of the IEEE Computer \& Communications Societies. IEEE. https://doi.org/10.1109/INFCOM.2000.832201

[4] Jukan, A., Nguyen, H. N., \& Van As, H. R. (2000). An approach to QoS-based routing for LEO satellite networks. International Conference on Communication Technology. IEEE. https://doi.org/10.1109/ICCT.2000.889340

[5] Tarik, T., Daisuke, M., \& Jamalipour, A. (2009). Explicit load balancing technique for NGEO satellite IP networks with on-board processing capabilitie. IEEE/ACM Trans. on Networking, 17(1), 281-293. https://doi.org/10.1109/TNET.2008.918084

[6] Rao, Y. \& Wang, R. C. (2010). Agent-based load balancing routing for Leo satellite networks. Computer Networks, 54(17), 3187-3195. https://doi.org/10.1016/..comnet.2010.06.019

[7] Ekici, E., Akyildiz, I. F., \& Bender, M. D. (2002). A distributed routing algorithm for datagram traffic in Leo satellite networks. IEEE/ACM Transactions on Networking, 9(2), 137-147. https://doi.org/10.1109/90.917071

[8] Cao, J. \& Stefanovic, M. (2010). Cross Entropy Accelerated Ant Routing in satellite networks. American Control Conference. IEEE. https://doi.org/10.1109/ACC.2010.5530698

[9] Werner, M. (1997). A dynamic routing concept for ATMbased satellite personal communication networks. IEEE Journal on Selected Areas in Communications, 15(8), 16361648. https://doi.org/10.1109/49.634801
[10] Ekici, E., Akyildiz, I. F., \& Bender, M. D. (2002). A distributed routing algorithm for datagram traffic in LEO satellite networks. IEEE/ACM Transactions on Networking, 9(2), 137-147. https://doi.org/10.1109/90.917071

[11] Yukio Hashimoto, B. S. (1998). Design of IP-based Routing in a LEO Satellite Network. Proc of Third International Workshop on Satellite-based Information Services.

[12] Lu, Y., Zhao, Y. J., Sun, F. C., Li, H. B., Ni, G. Q., \& Wang, D. J. (2014). Routing techniques on satellite networks. Journal of Software, 25(5), 1085-1100. https://doi.org/10.13328/j.cnki.jos.004581

[13] Xi, Y., \& Yeh, E. M. (2008). Pricing, Competition, and Routing for Selfish and Strategic Nodes in Multi-Hop Relay Networks. INFOCOM 2008. The 27th Conference on Computer Communications. IEEE. IEEE. https://doi.org/10.1109/INFOCOM.2008.205

[14] Ng, S. K. \& Seah, W. K. G. (2008). Game-Theoretic Model for Collaborative Protocols in Selfish, Tariff-Free, Multihop Wireless Networks. Infocom the Conference on Computer Communications IEEE. IEEE. https://doi.org/10.1109/INFOCOM.2008.52

[15] Keränen, A., Ott, J., \& Kärkkäinen, T. (2009). The ONE Simulator for DTN Protocol Evaluation. International Conference on Simulation Tools \& Techniques. ICST (Institute for Computer Sciences, Social-Informatics and Telecommunications Engineering). https://doi.org/10.4108/ICST.SIMUTOOLS2009.5674

[16] Vahdat, A., Becker, D., et al. (2000). Epidemic routing for partially connected ad hoc networks. Technical Report CS200006, Duke University.

[17] Balasubramanian, A., Levine, B., \& Venkataramani, A. (2007). Dtn routing as a resource allocation problem. $A C M$ SIGCOMM Computer Communication Review, 37(4), 373. https://doi.org/10.1145/1282427.1282422

\section{Contact information:}

\section{Ligang CONG}

(Corresponding author)

School of Computer Science and Technology,

Changchun University of Science and Technology,

Changchun 130022, China

E-mail: clg_cust@126.com

\section{Huamin YANG}

School of Computer Science and Technology,

Changchun University of Science and Technology,

Changchun 130022, China

E-mail: yhm@cust.edu.cn

\section{Yanghui WANG}

School of Artificial Intelligence,

Changchun University of Science and Technology,

Changchun 130022, China

E-mail: wyh@cust.edu.cn

\section{Xiaoqiang D}

School of Computer Science and Technology,

Changchun University of Science and Technology,

Changchun 130022, China

E-mail: dixiaoqiang@126.com 